\title{
Can the Pick-up Resonances help to Control the COVID-19 Virus?
}

\author{
Chi Yu Hu* \\ Department of Physics and Astronomy, California State University, USA \\ *Corresponding author: Chi Yu Hu, Department of Physics and Astronomy, California State University, USA
}

\begin{tabular}{|c|c|}
\hline ARTICLE INFO & ABSTRACT \\
\hline & $\begin{array}{l}\text { Citation: Chi Yu Hu. Can the Pick-up Resonances help to Control the COVID-19 Virus?. } \\
\text { Biomed J Sci \& Tech Res 33(2)-2021. BJSTR. MS.ID.005370. }\end{array}$ \\
\hline
\end{tabular}

\section{Short Communication}

The pick-up resonance, also known as Gailitis resonance, were unexpectantly uncovered six years ago from a high accuracy calculation with six open channels. The positron scattering with Hydrogen at energy slightly above positronium Ps $(n=2)$ formation threshold revealed the simple Stark-effect induced universal physical mechanism. Such classes of resonances can be found in any system where a charged group moves towards another polarizable group. For the 3-body scattering system mentioned above, that gives the atoms $\mathrm{H}$ or Ps electric dipole moments. Even the ground states or the S-states, their spherically symmetric wave functions will be distorted with an incoming charged object. Our calculations show, in the first case, the dipole moments give the scattering system an attractive potential energy proportional to $-1 / \mathrm{y}^{2}$. In the second case, when the atom is in a ground state, the attractive potential energy is proportional to $-1 / \mathrm{y}^{4}$, where $\mathrm{y}$ is the distance between the center of masses. All cases of the pick-up resonances have one thing in common. Their whole lifetime consists of the nature's pick-up action, there are specific goals for each such action afterwards. This physical mechanism has explained a number of decades old puzzles already. One of them is low energy nuclear fusion.

\section{The Physics of the COVID-19 Vaccine}

The only thing we need to know is the job of the complex unit named mRNA. Originally, they are constructed in the cells, their jobs are producing proteins of all kinds. When they are out of the cells, they can attach to the virus and produce those spike proteins on the surface of the virus. Such spike proteins will guide the virus back into the cells and destroy the whole cell. This kind of mRNA is now produced in the laboratory and used as a vaccine. Why? Because this lab mRNA produces different spike proteins. Instead of guiding the virus into the cell, they attract antibody and immune response to eliminate the virus. That is the current idea. From the Physics point of view, the mRNA is one of two hard working groups in the living body. The other is the enzymes group. Both are built in with electric charge to enable them working in their different working areas. The vaccine mRNA is not different. They are protein makers need to find a virus to make spike protein. In addition, this virus has spherical symmetry just like the ground (S-state) case discussed previously, where the polarizability of the ground state produces an attractive potential energy $\sim-1 / r^{4}$. Such resonances are formed very close to the virus, but the spike protein produced on the surface of the virus are different, they attract antibody and immune responses that lead to the destruction of the virus. The mRNA vaccine does not need to go in and out of the cell to be enabled to do the work, rather the universal physical mechanism induced by the Stark-effect produce pick-up resonances to do the job with its own electric field completely outside the cells. Some three-body theoretical tool can be found in references below. More complex system needs additional work to introduce the polarizability. These classes of systems belong to low energy quantum three body scattering system [1-3].

The low energy quantum 3-body scattering system is exactly soluble. the complete solution, including all resonances will be obtained. In principle, any of the resonant energies could be used to kill the virus. Armed with the recent super- power of supercomputers, any virus has no place to hide. That date should have come sooner. However, with the author's very limited knowledge of the complicated Biology processes, consider this 
note a grandmother's advice. What we have uncover so far from the three body system is only the tip of the ice-berg. The remaining work is waiting for you to endear and to enjoy.

\section{Acknowledgement}

I would like to thank Mark McLaughlin for his assistance.

ISSN: 2574-1241

DOI: 10.26717/BJSTR.2021.33.005370

Chi Yu Hu. Biomed J Sci \& Tech Res

(c) (P) This work is licensed under Creative BY Commons Attribution 4.0 License

Submission Link: https://biomedres.us/submit-manuscript.php

\section{References}

1. Hu CY (2016) The Faddeev - Merkuriev Differential Equations (MFE) And Multichannel 3-Body Scattering Systems. Atoms 4(2): 16.

2. Hu CY, Papp Z (2016) Second Order Stark-Effect Induced Gailitis Resonances ine + Ps and p + 7Li. Atoms 4(1): 8.

3. Hu CY, Caballero D (2020) Nature's Pick-up Tool, the Stark-Effect Induced Gailitis Resonances and Applications. Atoms 8(3): 32.

$\begin{array}{ll}\text { BIOMEDICAL } & \text { Assets of Publishing with us } \\ \text { RESEARCHES } & \text { - Global archiving of articles } \\ \text { - Immediate, unrestricted online access } & \text { - Rigorous Peer Review Process } \\ & \text { - Authors Retain Copyrights }\end{array}$

